\title{
Combined heart-kidney transplant improves post-transplant survival compared with isolated heart transplant in recipients with reduced glomerular filtration rate: Analysis of 593 combined heart-kidney transplants from the United Network Organ Sharing Database
}

Tara Karamlou, MD, ${ }^{a}$ Karl F. Welke, MD, ${ }^{\mathrm{b}}$ D. Michael McMullan, MD, ${ }^{\mathrm{b}}$ Gordon A. Cohen, MD, ${ }^{\mathrm{a}}$ Jill Gelow, MD, ${ }^{\mathrm{c}}$ Frederick A. Tibayan, MD, ${ }^{\mathrm{d}}$ James M. Mudd, MD, ${ }^{\mathrm{c}}$ Matthew S. Slater, MD, ${ }^{\mathrm{d}}$ and Howard K. Song, MD

Objective: Criteria for simultaneous heart-kidney transplant (HKTx) recipients are unclear. We characterized the evolution of combined HKTx in the United States over time compared with isolated heart transplantation (HTx) and determined factors maximizing post-transplant survival. We focused on whether a threshold estimated glomerular filtration rate (eGFR) could be identified that justified combined transplantation.

Methods: A supplemented United Network Organ Sharing Dataset identified HTx and HKTx recipients from 2000 to 2010. eGFR was calculated for HTx and recipients were grouped into eGFR quintiles. Time-related mortality was compared among recipients, with multivariable factors sought using Cox proportional hazard regression models.

Results: We identified 26,183 HTx recipients, of whom 593 were HKTx recipients. HTx increased modestly over time $(3.6 \%)$, whereas prevalence of HKTx increased dramatically $(147 \%)$. Risk-unadjusted survival was similar among HTx recipients $(8.4 \pm 0.04$ years $)$ and HKTx recipients $(7.7 \pm 0.2$ years $)(P=.76)$. Isolated HTx recipients in the lowest eGFR quintile had decreased survival $(P<.001)$, but those in the third eGFR quintile had superior survival, suggesting a benefit in this subgroup. HTx recipients in the lowest eGFR quintile (eGFR less than mean $37 \mathrm{~mL} /$ minute) had worse survival than combined HKTx recipients ( $7.1 \pm 0.07$ vs $7.7 \pm 0.2 ; P<.001)$. Multivariable factors for increased mortality among HTx recipients included lower eGFR, higher recent panel reactive antibody score, older age, African American race, diabetes, longer ischemic time, and certain diagnoses.

Conclusions: Performance of combined HKTx is increasing out of proportion to isolated HTx. eGFR is an important determinant of improved HTx survival. Combined HKTx recovers post-transplant survival in patients with eGFR $<37 \mathrm{~mL} /$ minute and can be recommended in this subgroup. (J Thorac Cardiovasc Surg 2014;147:456-61)

\footnotetext{
From the Division of Pediatric Cardiothoracic Surgery, ${ }^{a}$ Benioff Children's Hospital, University of California, San Francisco, San Francisco, Calif; Congenital Cardiac Center,' Department of Pediatric Cardiac Surgery, Seattle Children's Hospital, Seattle, Wash; Division of Cardiovascular Medicine, ${ }^{\mathrm{c}}$ Oregon Health \& Science University, Portland, Ore; and Division of Cardiothoracic Surgery, ${ }^{\mathrm{d}}$ Oregon Health \& Science University, Portland, Ore

Disclosures: Authors have nothing to disclose with regard to commercial support. Read at the 39th Annual Meeting of The Western Thoracic Surgical Association, Coeur d'Alene, Idaho, June 26-29, 2013.

Received for publication June 25, 2013; revisions received Sept 3, 2013; accepted for publication Sept 10, 2013; available ahead of print Nov 1, 2013

Address for reprints: Tara Karamlou, MD, Division of Pediatric Cardiothoracic Surgery, Benioff Children's Hospital, University of California San Francisco, 513 Parnassus Ave, Ste S-549, San Francisco, CA 94143 (E-mail: tara. karamlou@ucsfmedctr.org).

$0022-5223 / \$ 36.00$

Copyright (c) 2014 by The American Association for Thoracic Surgery

http://dx.doi.org/10.1016/j.jtcvs.2013.09.017
}

The first report of simultaneous combined heart and kidney transplantation (HKTx) using the same donor was by Norman and colleagues in 1978. ${ }^{1}$ Combined organ transplantation has since become an accepted therapy for combined kidney and cardiac failure, which frequently exist together. Although several single-institution studies have been published, ${ }^{2-4}$ contemporary national outcomes for HKTx recipients are not known. Additionally, there are no uniformly agreed upon criteria for selecting a combined organ transplant strategy over sequential or isolated organ transplant. We sought to characterize the evolution of HKTx relative to isolated heart transplantation $(\mathrm{HTx})$ in the United States and determine those factors that maximize posttransplant survival. We were particularly interested in whether a threshold estimated glomerular filtration rate (eGFR) could be identified that justified combined transplantation.

\section{METHODS}

All HTx and HKTx recipients from 2000 to 2010 were identified using a supplemented United Network for Organ Sharing (UNOS) dataset, termed 


\section{Abbreviations and Acronyms}

eGFR = estimated glomerular filtration rate

HKTx $=$ combined heart-kidney transplant

HTx $=$ heart transplant

LVAD $=$ left ventricular assist device

UNOS $=$ United Network for Organ Sharing

UNOS-Standard Transplant Analysis and Research. All HKTx procedures were performed either as simultaneous procedures, or performed sequentially within 24 hours of 1 another. The eGFR among HTx recipients was estimated using the widely accepted 4-variable Modification of Diet in Renal Disease formula for adults ${ }^{5-7}$ and the Schwartz formula for recipients younger than age 13 years. ${ }^{8,9}$ The 4-Modification of Diet in Renal Disease formula incorporates serum creatinine, age, race, and sex, and has been validated for patients with chronic renal disease. The Schwartz formula (eGFR $=[k \cdot$ height/serum creatinine $]$ ), includes a constant, $k$, that varies with age to account for variable muscle mass. The serum creatinine used in all calculations was obtained at the most proximate measurement before transplantation. The eGFR was then log-transformed and recipients were grouped into eGFR quintiles. Continuous variables are reported as mean \pm standard deviation, or as medians with range, and are compared across groups using the Student $t$ test (for normal distribution) or the Wilcoxon rank sum test (for non-normal distribution). Comparisons among categorical variables are presented as percentages and frequencies and were compared across groups using the $\chi^{2}$ test or Fisher exact test as appropriate. The prevalence of HKTx compared with isolated HTx over time was calculated for each year of the study period. Time-related survival from HTx or HKTx were described using the Kaplan-Meier method, and risk-unadjusted differences among groups were determined using the log-rank test. Particular attention was directed at investigating an eGFR threshold at which combined HKTx survival provided a benefit over isolated HTx. Multivariable factors for time-related death were sought using Cox proportional hazard regression models. Variables considered in multivariable models are reported in Table E1. In all cases, variable transformations were explored and optimized to provide optimum calibration, and year was included in all models to account for a possible era effect. SAS software, version 9.2 (SAS Institute Inc, Cary, NC) was used for all analyses. Institutional review board approval was obtained under an expedited review for de-identified existing data.

\section{RESULTS}

\section{Patient Characteristics}

We identified 26,183 HTx recipients, of whom 593 were HKTx recipients. Although the frequency of isolated HTx increased modestly over time $(3.6 \%)$, the prevalence of HKTx increased dramatically $(147 \%)$ over time (Figure 1, $A$ and $B$ ). Demographics and clinical characteristics of the study patients segregated by recipient type are shown in Table 1 . The median patient age was 52 years (range 0-77 years). HKTx recipients had higher serum creatinine at the time of transplant $(3.4 \pm 2.8 \mathrm{mg} / \mathrm{dL}$ vs $1.2 \pm 0.7 \mathrm{mg} / \mathrm{dL})$, were older $(51 \pm 13$ years vs $45 \pm 20$ years), and had a higher prevalence of diabetes $(40 \%$ vs $36 \%)$, compared with HTx recipients $(P<.001$ for all). A lower proportion of HKTx recipients were on left ventricular assist device (LVAD) support compared with HTx recipients ( $15.2 \%$ vs $20.4 \% ; P=.003$ ), although other types of inotropic or mechanical circulatory support were similar among both groups. Wait-list times were not significantly different among recipient groups (HTx: $193 \pm 351$ days vs HKTx: $212 \pm 322$ days; $P=.14$ ).

Among isolated HTx recipients, the mean eGFR for grouped quintiles in ascending order was: $36.6 \pm 8.9 \mathrm{~mL} /$ minute, $54.8 \pm 4.1 \mathrm{~mL} /$ minute, $68.9 \pm 4.2 \mathrm{~mL} / \mathrm{minute}$, $87.1 \pm 6.6 \mathrm{~mL} /$ minute, and $140.3 \pm 56.4 \mathrm{~mL} / \mathrm{minute}$, respectively. eGFR was negatively correlated with diabetes, white ethnicity, being a man, and age. The most prevalent diagnoses overall included dilated cardiomyopathy $(72 \%)$, ischemic cardiomyopathy $(5 \%)$, and congenital heart disease $(7 \%)$. The majority of patients were white $(70 \%)$ and men $(73 \%)$.

\section{Renal Failure Requiring Dialysis Among Recipients}

Following transplant, $155(26 \%)$ of the combined HKTx recipients required dialysis within 30 days, compared with $2181(9 \%)$ of the isolated HTx recipients. Among isolated HTx recipients, $609(22 \%)$ of 2758 patients in the lowest GFR quintile required early dialysis, compared with $16 \%$ (1115 out of 6962 ) and $11 \%$ (1836 out of 15781) of patients in the next 2 quintiles. Logistic regression analysis of patients receiving isolated heart transplant, including renal function indices ( $c$ statistic, 0.67$)$ revealed that a higher eGFR reduced the risk of post-transplant early dialysis (odds ratio, 0.647; 95\% confidence interval, 0.574-0.72; $P<.001)$ Patients in the lowest eGFR quintile had a significantly increased risk of needing dialysis (odds ratio, 2.093; 95\% confidence interval, 1.835-2.386; $P<.001$ ).

\section{Influence of eGFR on Post-Transplant Survival}

Overall survival at 1 and 5 years for the entire cohort was $88 \%$ and $73 \%$, respectively. Risk-unadjusted survival was similar among HTx $(8.4 \pm 0.04$ years $)$ and HKTx recipients $(7.7 \pm 0.2$ years; $P=.76$ ) (Figure 2). Isolated HTx recipients in the lowest eGFR quintile had significantly decreased survival compared with the other HTx groups $(P<.001)$, whereas those in the third eGFR quintile had superior survival, suggesting a benefit in this subgroup (Figure 3).

Compared with patients receiving combined HKTx, isolated HTx recipients in the lowest eGFR quintile (mean eGFR $<37 \mathrm{~mL} /$ minute) had significantly worse median survival $(7.1 \pm 0.07$ years vs $7.7 \pm 0.2$ years; $P<.001$ ) (Figure 4). Multivariable analysis demonstrated that lower eGFR (log-transformed; hazard ratio, 0.83; $P<.001$ ), higher recent panel reactive antibody (hazard ratio, 1.001; $P<.001$ ), older age (hazard ratio, 1.002; $P=.03$ ), African American race (hazard ratio, 1.55; $P<.001$ ), diabetes (hazard ratio, $1.17 ; P<.001$ ), longer ischemic time (hazard ratio, 1.08; $P<.001$ ), and certain diagnoses were risk factors for increased mortality among HTx recipients (Table E2). 


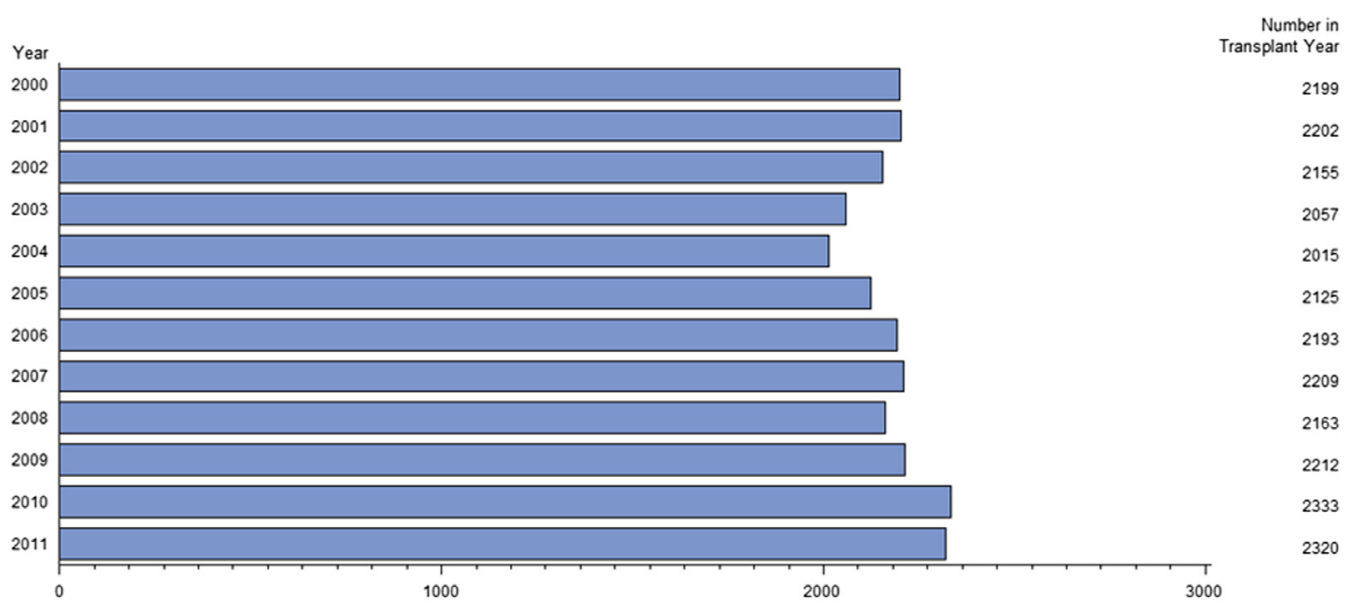

A

Number of Isolated Heart Transplants

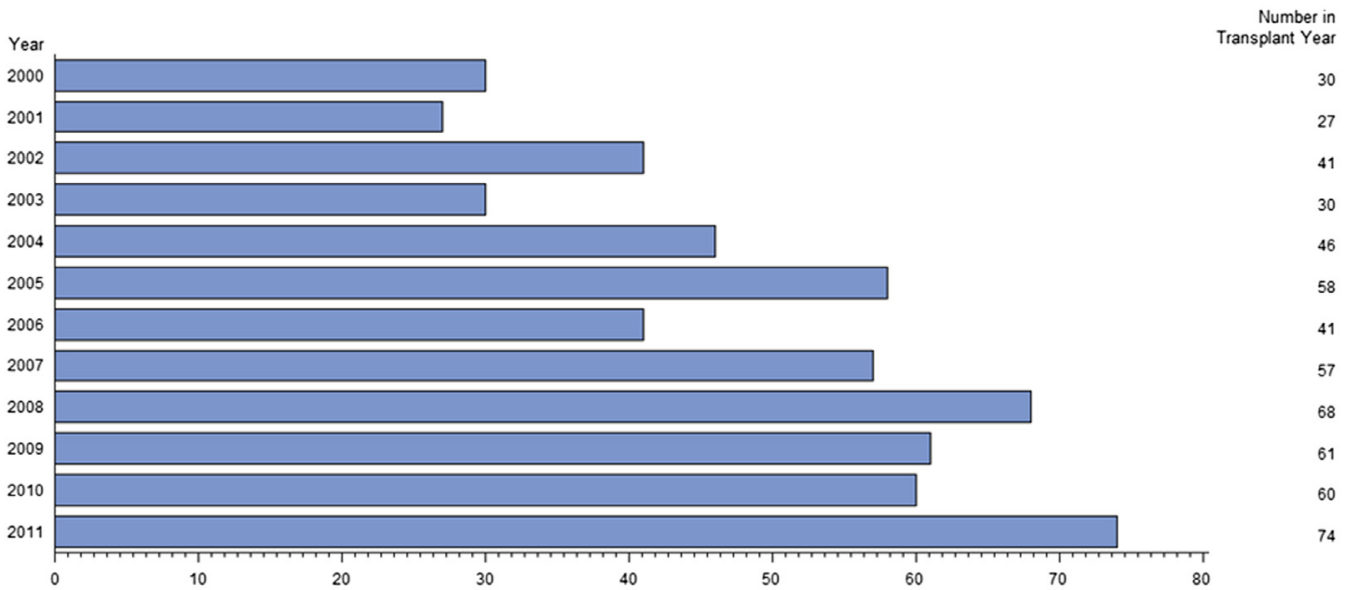

B

Number of Heart-Kidney Transplants

FIGURE 1. A, Horizontal bar graph depicting the number of isolated heart transplants performed in each year of the study period. Actual numerical value depicted on the right side, with calendar year on the left side. B, Horizontal bar graph depicting the number of combined heart-kidney transplants performed in each year of the study period. Actual numerical value depicted on the right side, with calendar year on the left side.

\section{DISCUSSION}

Our study is the first to report contemporary national outcomes for combined HKTx in relation to isolated HTx. In addition, our study provides useful criteria, based on the eGFR at the time of transplantation, for determining when a combined transplant strategy would be advantageous. Central to a discussion of combined organ transplantation is the question of whether native renal function will improve following heart transplantation alone. Although our study cannot provide a definitive answer to this important question, we do provide data suggesting that early renal insufficiency requiring dialysis is relatively frequent among HTx recipients, and that reduced eGFR, especially below a threshold of $37 \mathrm{~mL} /$ minute, increases this risk. We also found that despite only a modest increase in the number of isolated HTx procedures performed yearly, combined HKTx rates are rising more rapidly. It is likely, therefore, that initiatives to develop evidence-based guidelines for multiorgan allocation are timely, and might be particularly beneficial.

Our recently published article ${ }^{10}$ demonstrated that LVAD use might optimize outcomes among HTx recipients compared with other types of mechanical circulatory support. Our study described here demonstrated a benefit, as expected, among those recipients who did not require either inotropic nor mechanical circulatory support as well as a disadvantage to those patients requiring use of intra-aortic balloon pump immediately before transplantation. However, there was no benefit seen among recipients managed with LVAD versus other types of assist devices. As elucidated in our prior manuscript, the UNOS dataset may not provide enough granularity at present to distinguish among types of LVAD device and UNOS also does not allow determination of the timing of LVAD 
TABLE 1. Patient characteristics segregated by recipient type

\begin{tabular}{lccc}
\hline \multicolumn{1}{c}{ Variable } & $\begin{array}{c}\text { Combined } \\
\text { heart-kidney } \\
\text { transplant } \\
(\mathbf{n}=\mathbf{5 9 3})\end{array}$ & $\begin{array}{c}\text { Isolated heart } \\
\text { transplant } \\
(\mathbf{n}=\mathbf{2 5 , 5 9 0})\end{array}$ & $\boldsymbol{P}$ \\
\hline Age, y & $51.1 \pm 13.4$ & $45.2 \pm 20$ & $<.001$ \\
Weight, kg & $79.1 \pm 19.8$ & $79.9 \pm 26.9$ & .41 \\
Creatinine at Tx, mg/dL & $3.43 \pm 2.80$ & $1.21 \pm 0.79$ & $<.001$ \\
Women & $133(22)$ & $7047(28)$ & .006 \\
Status 1A & $109(18)$ & $6771(26)$ & $<.001$ \\
Diabetes & $214(36)$ & $5074(20)$ & $<.001$ \\
African American & $148(25)$ & $4423(17)$ & $<.001$ \\
Days on waitlist & $95(1-2824)$ & $72(0-5815)$ & .14 \\
Ischemic time, h & $3.2 \pm 1.1$ & $3.3 \pm 1.1$ & .04 \\
Underlying diagnosis & & & \\
$\quad$ Dilated CM & $377(63)$ & $18,635(72)$ & $<.001$ \\
Ischemic CM & $25(4)$ & $1033(4)$ & .82 \\
CHD & $13(2)$ & $2071(8)$ & $<.001$ \\
$\quad$ Other & $178(29)$ & $3851(16)$ & $<.001$ \\
LVAD support at Tx & $90(15)$ & $5219(20)$ & .003 \\
Inotropic support at Tx & $224(38)$ & $9298(36)$ & .75 \\
IABP at Tx & $26(4)$ & $901(4)$ & .58 \\
BiVAD support at Tx & $11(2)$ & $623(2)$ & .31 \\
RVAD support & $2(.3 \%)$ & $54(.2 \%)$ & .51 \\
\hline Var &
\end{tabular}

Values are presented as mean \pm standard deviation or medians (range); frequencies are presented as n (\%). Tx, Transplant; $C M$, cardiomyopathy; $C H D$, congenital heart disease; $L V A D$, left ventricular assist device; IABP, intra-aortic balloon pump; $B i V A D$, biventricular assist device; $R V A D$, right ventricular assist device.

implementation. Further studies using a dedicated dataset, such as the Interagency Registry for Mechanically Assisted Circulatory Support, would be insightful.

Narula and colleagues ${ }^{11}$ published the only other national study of combined HKTx in 1997, using the UNOS. ${ }^{11}$ That study used data from 1987-1995, circumscribing the period that included UNOS registry inception. Eighty-four HKTx recipients were compared with 14,340 isolated HTx recipients. In agreement with our study, overall survival was equivalent among both groups.

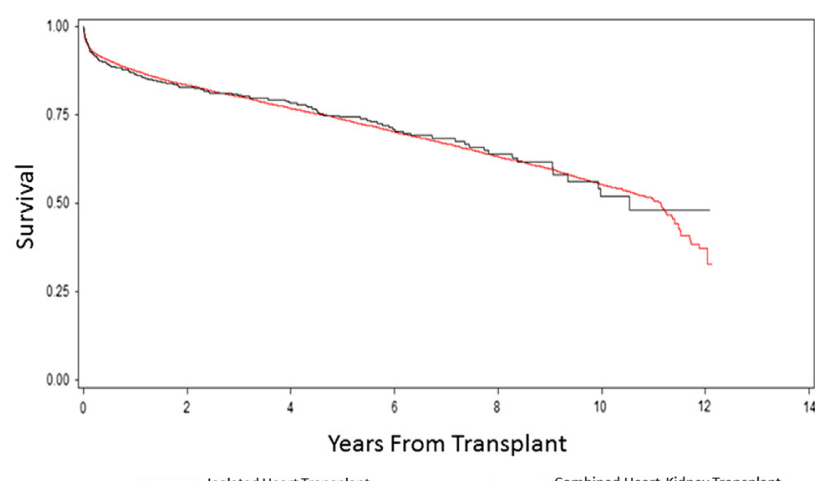

FIGURE 2. Kaplan-Meier plot depicting post-transplant survival among isolated heart transplant recipients (red line) compared with post-transplant survival among combined heart-kidney transplant recipients. Note that survival is equivalent among both groups.
As has been subsequently shown in other studies, ${ }^{12-14}$ combined transplantation reduced the prevalence of cardiac allograft rejection. Although the putative mechanism of reduced allograft rejection (both kidney and heart) remains unclear, it is speculated that the organ combination induces immunogenic tolerance. ${ }^{15}$ The study by Narula et al ${ }^{11}$ did not report outcomes other than survival or allograft rejection, and did not stratify patients based on pretransplant renal function.

Multiple single-institution studies of combined HKTx have been reported. ${ }^{2-4,14,16,17}$ Raichlin and colleagues ${ }^{4}$ compared the results of 12 patients undergoing combined organ transplantation with those of 183 patients undergoing isolated HTx at the Mayo Clinic over a 10-year period (1996-2007). Post-transplant survival was equivalent in both groups, as in our study described here. Importantly, although their numbers were small, they showed that kidney allograft function at 1-year, as measured by GFR, remained superior to native renal function among isolated HTx recipients. Although the study by Raichlin and colleagues ${ }^{4}$ did not stratify patients by pre-transplant GFR, in a subgroup of 19 isolated HTx recipients with GFR $<40 \mathrm{~mL} /$ minute, 7 (44\%) developed end-stage renal failure and $4(25 \%)$ underwent kidney transplantation within 3 years. The contention that preoperative renal function predicts the development of chronic renal insufficiency following HTx is supported by data from both Vossler and colleagues $^{18}$ and Al Aly and colleagues. ${ }^{19}$ The study by Vossler and colleagues ${ }^{18}$ established a mean creatinine threshold of $1.6 \mathrm{mg} / \mathrm{dL}$ as a risk factor for chronic renal insufficiency, whereas Al Aly and colleagues ${ }^{18}$ showed a predictable decrement in renal function of $10 \mathrm{~mL} /$ minute/ $1.73 \mathrm{~m}^{2}$ at 10 and 15 years as well as an increased mortality in survivors of HTx procedure. Multiple factors contribute to decreased renal function among HTx recipients, including nephrotoxic immunosuppressive medications, compromised cardiac allograft function, infection, and exacerbation of underlying comorbidities. In our study, there was an important prevalence $(9 \%)$ of early post-transplant dialysis among isolated HTx recipients, and the risk of early dialysis was higher among recipients with impaired renal function.

Czer and colleagues ${ }^{2}$ reviewed their single institution outcomes for 30 patients undergoing combined HKTx compared with 440 patients undergoing isolated HTx. They also showed similar survival among both recipient groups. Although they reported that renal function remained equivalent among the combined recipients and the isolated HTx recipients, there was still an $8.5 \%$ incidence of renal failure among those receiving only cardiac allografts.

A strength of our study is the stratification of transplant candidates based on a reproducible and objective measure of renal function, and the use of this marker, the eGFR, to develop criteria in which combined transplantation is 


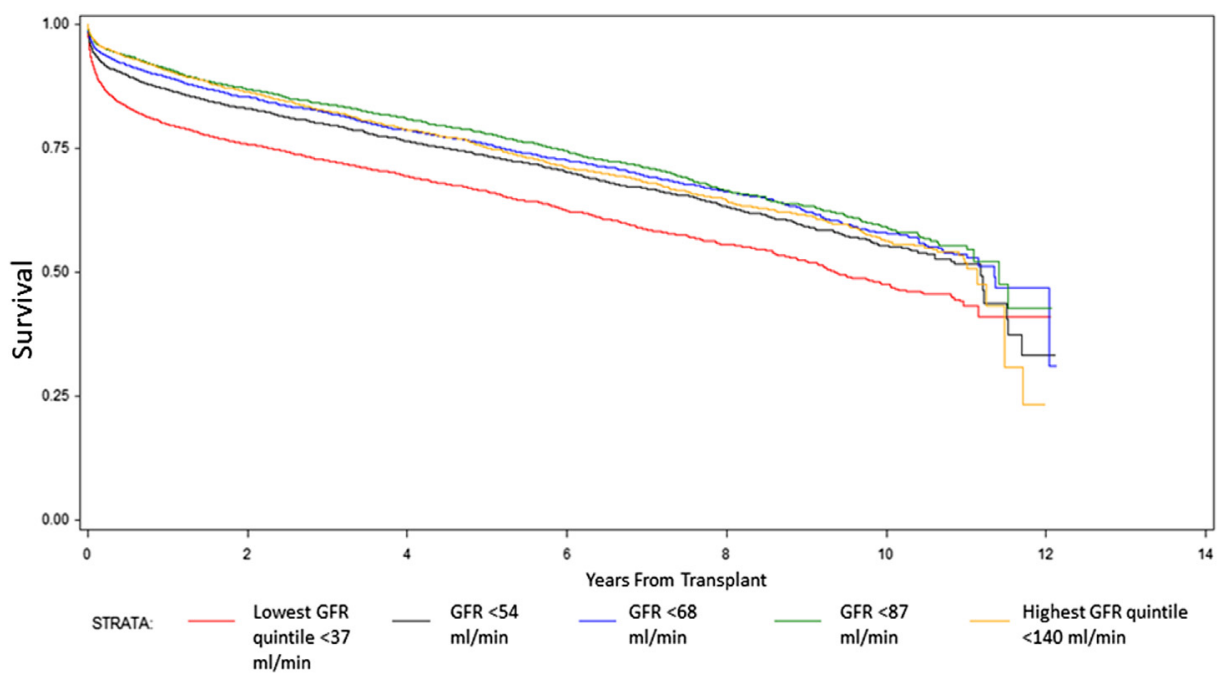

FIGURE 3. Kaplan-Meier plot depicting post-transplant survival among isolated heart-transplant recipients stratified by glomerular filtration rate (GFR) quintiles. Note that recipients in the lowest GFR quintile (GFR $<37 \mathrm{~mL} /$ minute [red line]) had significantly lower survival compared with the other 4 groups.

beneficial. Stratification was possible because of the large number of patients included in the UNOS registry. Although others have proposed criteria, the predictions are limited by small numbers of patients. Savdie and colleagues $^{20}$ suggested a threshold of GFR $<30 \mathrm{~mL} /$ minute based on 7 patients, whereas Smith and colleageus ${ }^{21}$ suggested that creatinine $<2 \mathrm{mg} / \mathrm{dL}$ be used for cardiac retransplant candidates with cyclosporine-induced nephrotoxicity based on 66 patients. Clearly, additional studies are needed to refine our proposed criteria and validate them in a prospective cohort of patients.

\section{Limitations}

Our study is a retrospective review with limited information regarding preclinical status and timing of interventions. Unfortunately, in 11,261 patients the

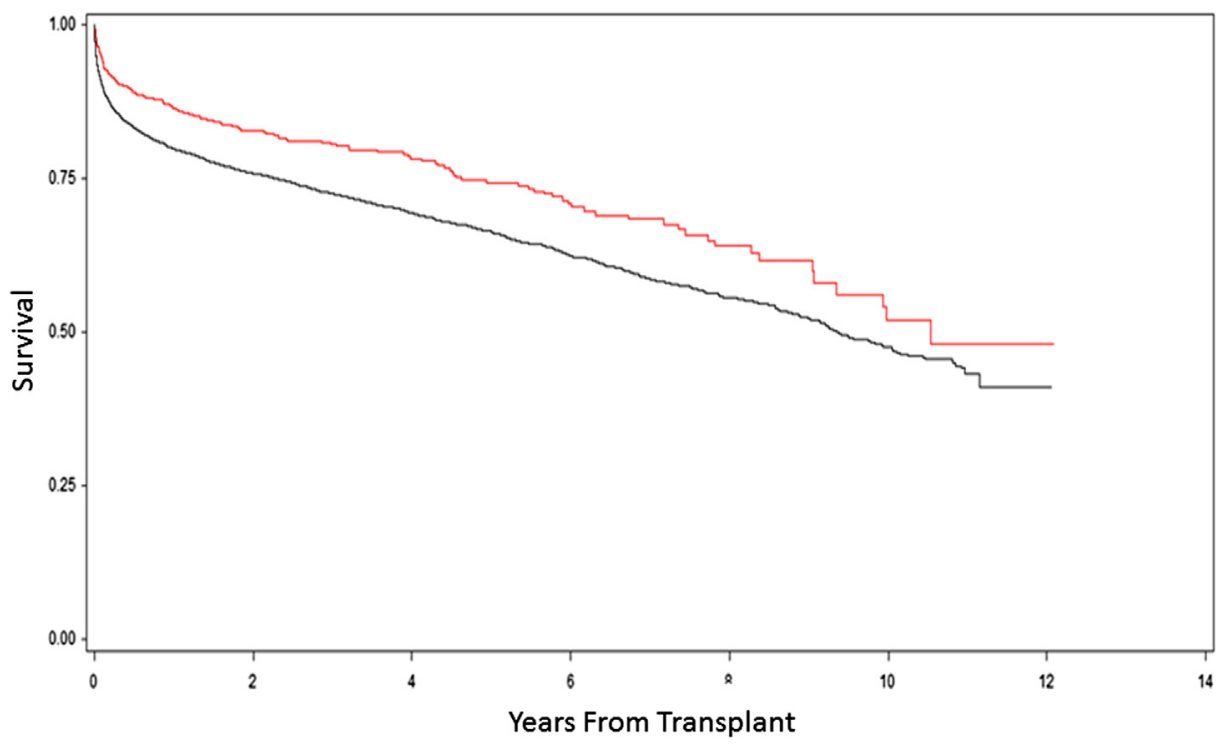

Combined Heart-Kidney Transplant $\quad \begin{gathered}\text { Isolated Heart Transplant } \\ \text { Recipients with GFR }<37 \mathrm{ml} / \mathrm{min}\end{gathered}$

FIGURE 4. Kaplan-Meier plot depicting the post-transplant survival among isolated heart transplant recipients in the lowest glomerular filtration rate $($ GFR) quintile (GFR $<37 \mathrm{~mL} /$ minute [black line]) compared with post-transplant survival among combined heart-kidney transplant recipients (red line). Although overall survival was equivalent among both isolated heart transplant and combined organ transplant recipients, there is a significant survival advantage when a combined transplant strategy is used compared with isolated heart transplant in the subgroup of patients with GFR $<37 \mathrm{~mL} / \mathrm{minute}$. 
presence or absence of renal replacement therapy was not documented and therefore it was not possible to include these patients in the analysis. Specific pretransplant risk factors that could be informative regarding outcome, including peripheral vascular disease and functional status, were also missing in large numbers of patients $(\mathrm{n}=11,267)$.

Post-transplant immunosuppression regimens are different among recipient groups, with the combined organ recipients likely receiving more aggressive therapy. If this therapy favorably biased survival toward the combined organ recipients is unknown, although equivalent survival has been reported by multiple other groups, some of whom adjusted for different postoperative immunosuppression. Although immunosuppression medications are available as variables in the general UNOS registry, these data were not included in the specialized dataset used for our analysis.

Our study was focused on survival and early renal failure. We did not investigate graft rejection and cannot provide any information about whether or not allograft rejection was accelerated in either group.

\section{CONCLUSIONS}

HKTx is increasing as a percentage of total HTx. Pretransplant eGFR is an important determinant of improved HTx recipient survival, and can also predict the occurrence of postoperative renal failure. Combined organ transplantation recovers post-transplant survival in patients with eGFR $<37 \mathrm{~mL} /$ minute and can be recommended for potential HTx candidates in this subgroup.

\section{References}

1. Norman JC, Brook MI, Cooley DA, Kilma T, Kahan BD, Frazier OH, et al. Total support of the circulation of a patient with post-cardiotomy stone-heart syndrome by a partial artificial heart (ALVAD) for 5 days followed by heart and kidney transplantation. Lancet. 1978;1:1125-7.

2. Czer LSC, Ruzza A, Vespignani R, Jordan S, De Robertis MA, Mirocha J, et al. Survival and allograft rejection rates after combined heart and kidney transplantation in comparison with heart transplantation alone. Transplant Proc. 2011;43:3869-76

3. Laufner G, Kocher A, Grabenwoger M, Berlakovich GA, Zuckermann A, Ofner P, et al. Simultaneous heart and kidney transplantation as treatment for end-stage heart and kidney failure. Transplantation. 1997;64:1129-34.

4. Raichlin E, Kushwaha SS, Daly RC, Kremers WK, Frantz RP, Clavell AL, et al, Combined heart and kidney transplantation provides excellent survival and decreases risk of cardiac cellular rejection and coronary allograft vasculopathy Transplant Proc. 2011:43:1871-6.

5. Levey AS, Bosch JP, Lewis JB, Greene T, Rogers N, Roth D. A more accurate method to estimate glomerular filtration rate from serum creatinine: a new prediction equation. Modification of Diet in Renal Disease Study Group. Ann Intern Med. 1999;130:461-70.

6. Mathew TH, Johnson DW, Jones GR. Chronic kidney disease and automatic reporting of estimated glomerular filtration rate: revised recommendations. Med J Aust. 2005;187:459-63.

7. Joint Specialty Committee on Renal Medicine of the Royal College of Physicians of London and the Renal Association. Chronic kidney disease in adults: UK guidelines for identification, management and referral. Available at: http://www.renal.org/ CKDguide/full/CKDprintedfullguide.pdf. Accessed October 13, 2013.

8. Schwartz GJ, Haycock GB, Edelmann CM, Spitzer A. A simple estimate of glomerular filtration rate in children derived from body length and plasma creatinine. Pediatrics. 1976;58:259-63.

9. Schwartz GJ, Feld LG, Langford DJ. A simple estimate of glomerular filtration rate in full-term infants during the first year of life. J Pediatr. 1984; 104:849-54.

10. Karamlou T, Gelow J, Tibayan F, Slater M, Mudd J, Song HKS Mechanical circulatory support pathways that maximize survival following heart transplantation. Ann Thorac Surg. 2013;145:470-5.

11. Narula J, Bennett LE, Disalvo T, Hosenpud JD, Semigran MJ, Dec GW Outcomes in recipients of combined heart-kidney transplantation: multiorgan, same-donor study of the International Society of Heart and Lung Transplantation/United Network for Organ Sharing Scientific Registry. Transplantation. 1997;63:861-7.

12. Trachiotis GD, Vega JD, Johnston TS, Berg A, Whelchel J, Smith AL, et al Ten-year follow-up in patients with combined heart and kidney transplantation. J Thorac Cardiovasc Surg. 2003;123:2065-71.

13. Blanche C, Valenza M, Czer LS, Barath P, Admon D, Harasty D, et al Combined heart and kidney transplantation with allografts from the same donor. Ann Thorac Surg. 1994;58:1135.

14. Kabschull L, Schleicher C, Palmes D, Sindermann J, Suwelack B, Senninger N, et al. Renal graft outcome in combined heart-kidney transplantation compared to kidney transplantation alone: a single-center, matched-control study. Thorac Cardiovasc Surg. 2012;60:57-63.

15. Castillo-Luga, Brinker JA, Karl R. An overview of combined heart and kidney trabsplantation. Curr Opin Cardiol. 1999;14:121.

16. Busson M, Benoit G, Loisance D, Castaing D, Martin X, N'Doye P, et al Long-term results of simultaneous multiple-organ transplants. Transplant Proc. 1995;27:2446-7.

17. Colucci V, Quaini E, Colombo T, Colombo T, De Carlis L, Grassi M, et al Combined heart and kidney transplantation: an effective option-report of six cases. Eur J Cardiothorac Surg. 1997;12:654-8.

18. Vossler MR, Ni H, Toy W, Hershberger RE. Pre-operative renal function predicts development of chronic renal insufficiency after orthotopic heart transplantation. J Heart Lung Transplant. 2002;21:874-81.

19. Al Aly Z, Abbas S, Moore E, Diallo O, Hauptman PJ, Bastani B. The natural history of renal function following orthotopic heart transplant. Clin Transplant 2005; 19:683-9.

20. Savdie E, Keogh A, Macdonald P, Spratt PM, Graham AM, Golovsky D, et al Simultaneous transplantation of the heart and kidney. Aust N Z J Med. 1994; 24:554-60.

21. Smith J, Ribakove G, Hunt S, Miller J, Stinson EB, Oyer PE, et al. Heart retransplantation: the 25-year experience at a single institution. J Heart Lung Transplant. 1995; 14:832-9. 
TABLE E1. Variables considered in multivariable models.

\section{Age, $y$}

Transplant weight, $\mathrm{kg}$

Creatinine at time of transplant

Estimated glomerular filtration rate (log-transformed)

Estimated glomerular filtration rate quintile

Waitlist time, $\mathrm{d}$

Highest panel reactive antibody level

Most recent panel reactive antibody level

Ischemic time, $\mathrm{h}$

Status at listing

Female sex

Race (ie, white, African American, Hispanic, Asian, other)

Blood group

Diabetes

Diabetes duration $>5 \mathrm{y}$

Transplant year

Inotropic or mechanical circulatory support types

Underlying diagnosis (eg, congenital heart disease, dilated

cardiomyopathy, restrictive cardiomyopathy)

TABLE E2. Multivariable factors among isolated heart transplant recipients that are associated with post-transplant mortality

\begin{tabular}{lccr}
\hline \multicolumn{1}{c}{ Variable } & $\begin{array}{c}\text { Hazard } \\
\text { ratio }\end{array}$ & $\begin{array}{c}\text { 95\% confidence } \\
\text { interval }\end{array}$ & \multicolumn{1}{c}{$\boldsymbol{P}$} \\
\hline $\begin{array}{l}\text { Most recent panel reactive } \\
\quad \text { antibody (per \%) }\end{array}$ & 1.004 & $1.002-1.006$ & $<.001$ \\
Woman & & & \\
No support at Tx & 1.084 & $1.025-1.146$ & .004 \\
IABP at Tx & 0.831 & $0.775-0.892$ & $<.001$ \\
African American race & 1.189 & $1.070-1.321$ & .001 \\
Dx of CHD & 1.537 & $1.446-1.634$ & $<.001$ \\
Dx of dilated CM & 1.295 & $1.170-1.433$ & $<.001$ \\
Dx of HCM & 0.878 & $0.824-0.934$ & $<.001$ \\
Ischemic CM & 0.757 & $0.613-0.8934$ & $<.001$ \\
Diabetes & 1.243 & $1.177-1.313$ & $<.001$ \\
Longer ischemic time, h & 1.167 & $1.100-1.239$ & $<.001$ \\
Blood type O & 1.080 & $1.056-1.105$ & $<.001$ \\
Most recent era & 1.093 & $1.041-1.147$ & $<.001$ \\
Combined HKTx & 0.878 & $0.820-0.941$ & $<.001$ \\
eGFR $<35 \mathrm{~mL} /$ min (lowest quintile) & 1.233 & $1.131-1.345$ & $<.001$ \\
eGFR $<50 \mathrm{~mL} / \mathrm{min}$ (second quintile) & 1.134 & $1.052-1.222$ & .001 \\
eGFR* & 0.812 & $0.760-0.869$ & $<.001$ \\
\hline
\end{tabular}

$T x$, Transplant; $I A B P$, intra-aortic balloon pump; $D x$, diagnosis; $C H D$, congenital heart disease; $C M$, cardiomyopathy; $H C M$, hypertrophic cardiomyopathy; HKTx, combined heart kidney transplant; $e G F R$, estimated glomerular filtration rate. *log transformed. 